\title{
The molecular basis for apoptotic defects in patients with CD95 (Fas/Apo-1) mutations
}

\author{
Akshay K. Vaishnaw, ${ }^{1}$ Jason R. Orlinick, ${ }^{2}$ Jia-Li Chu, ${ }^{1}$ Peter H. Krammer, ${ }^{3}$ \\ Moses V. Chao, ${ }^{4}$ and Keith B. Elkon ${ }^{1}$ \\ ${ }^{1}$ Hospital for Special Surgery, Cornell University Medical College, New York, New York 10021, USA \\ ${ }^{2}$ Cornell University Medical College, New York, New York 10021, USA \\ ${ }^{3}$ Institute for Immunology and Genetics, German Cancer Research Center, 69009 Heidelberg, Germany \\ ${ }^{4}$ Skirball Institute, New York University Medical Center, New York, New York 10016, USA \\ Address correspondence to: Keith B. Elkon, Research Division, Hospital for Special Surgery, \\ Cornell University Medical Center 535 East 70th Street, New York, New York 10021, USA. Phone: (212) 606-1409; \\ Fax: (212) 717-1192; E-mail: elkonk@hss.edu \\ Received for publication September 4, 1998, and accepted in revised form December 14, 1998.
}

\begin{abstract}
Heterozygous mutations of the receptor CD95 (Fas/Apo-1) are associated with defective lymphocyte apoptosis and a clinical disease characterized by lymphadenopathy, splenomegaly, and systemic autoimmunity. From our cohort of 11 families, we studied eight patients to define the mechanisms responsible for defective CD95-mediated apoptosis. Mutations in and around the death domain of CD95 had a dominant-negative effect that was explained by interference with the recruitment of the signal adapter protein, FADD, to the death domain. The intracellular domain (ICD) mutations were associated with a highly penetrant Canale-Smith syndrome (CSS) phenotype and an autosomal dominant inheritance pattern. In contrast, mutations affecting the CD95 extracellular domain (ECD) resulted in failure of extracellular expression of the mutant protein or impaired binding to CD95 ligand. They did not have a dominant-negative effect. In each of the families with an ECD mutation, only a single individual was affected. These observations were consistent with differing mechanisms of action and modes of inheritance of ICD and ECD mutations, suggesting that individuals with an ECD mutation may require additional defect(s) for expression of CSS.
\end{abstract}

J. Clin. Invest. 103:355-363 (1999)

\section{Introduction}

CD95 (Fas/Apo-1), a member of the nerve growth factor/tumor necrosis factor (NGF/TNF) receptor superfamily, initiates a signal-transduction cascade leading to programmed cell death. The extracellular portion of the CD95 receptor contains three cysteine-rich domains $(\mathrm{CRD} 1,2,3)$ that all appear to be necessary for CD95 ligand binding (1). The intracellular region of CD95 contains an 80-amino acid region called the death domain. This is a protein-protein association motif found in other proapoptotic receptors: TNF receptor I, DR 3-5, and in their associated binding or signal-transducing molecules FADD, TRADD, RAIDD, and RIP (reviewed in ref. 2). CD95 ligand induces clustering of CD95, which leads to recruitment of FADD (MORT1) to the receptor death domain $(3,4)$. This initiates an apoptotic program, mediated by activation of a family of proteases termed caspases (reviewed in ref. 5). CD95 aggregation is also associated with ceramide generation (6-8) and induces recruitment of Daxx (9) and a novel kinase activity (10) to the CD95 intracellular domain (ICD).

CD95 is expressed at high levels in activated lymphocytes and acts as a major pathway for the peripheral deletion of antigen-primed lymphocytes (2), including potentially autoreactive $\mathrm{T}$ and $\mathrm{B}$ cells (11-13). Mice with mutations of CD95 or CD95 ligand (CD95L) develop a syndrome characterized by lymphoproliferation (lpr) or generalized lymphadenopathy (gld), together with sys- temic autoimmunity (14). Humans with massive lymphadenopathy and autoimmunity were described by Canale and Smith in 1967 (15), and two of the original patients described were subsequently shown to have CD95 mutations (16). Others have identified patients with the lpr phenotype (17) and heterozygous CD95 mutations $(18,19)$. Dianzani et al. (20) have described patients with defective CD95-mediated apoptosis but who do not have CD95 mutations. These patients were assumed to have a defect distal to CD95 in the signaling pathway, evidenced by lymphocyte resistance to ceramide-induced apoptosis. At present, the reason why only a portion of CD95 mutation carriers within a pedigree develop clinical disease, and the molecular mechanisms responsible for defective CD95 signal transduction, are not understood.

At least 20 distinct CD95 mutations have been reported in humans $(16,18,19,21-24)$. The majority of these mutations $(14 / 20)$ were heterozygous and involved the CD95 ICD. In transfection studies, Fisher et al. (19) demonstrated that ICD mutant CD95 proteins had a dominant-negative effect on CD95-mediated apoptosis, although the exact molecular action of these mutations was not defined. However, a dominant-negative effect does not readily explain the apoptotic defect in $(6 / 20)$ patients who have mutations within the CD95 extracellular domain (ECD). We have recently identified another eight families with heterozygous CD95 mutations, including three 
Table 1

Clinical features of CSS index cases

\begin{tabular}{|c|c|c|c|}
\hline Patient & Gender & Age of onset (years) & Clinical syndrome \\
\hline P4 & Female & 2.5 & $\begin{array}{l}\text { Hemolytic anemia, lymphadenopathy, hepatosplenomegaly, recurrent } \\
\text { headaches, seizures, loss of consciousness, membranous nephropathy }\end{array}$ \\
\hline P5 & Male & Neonate & $\begin{array}{l}\text { Autoimmune hemolytic anemia }(\mathrm{AlHA}) \text {, thrombocytopenia, } \\
\text { lymphadenopathy, splenomegaly }(\mathrm{S})^{\mathrm{A}}\end{array}$ \\
\hline P6 & Male & 2 & $\begin{array}{l}\text { AlHA, thrombocytopenia, anti-cardiolipin antibodies, } \\
\text { urticarial rashes, lymphadenopathy, hepatosplenomegaly }\end{array}$ \\
\hline P7 & Male & Neonate & AlHA, thrombocytopenia, lymphadenopathy, hepatosplenomegaly \\
\hline P8 & Female & Neonate & Severe AIHA, neutropenia, thrombocytopenia, rashes (S) \\
\hline P9 & Female & Neonate & $\begin{array}{l}\text { AlHA, neutropenia, lymphopenia, lymphadenopathy, } \\
\text { recurrent painful oral ulceration, premature ovarian failure (S) }\end{array}$ \\
\hline P10 & Male & Neonate & $\begin{array}{l}\text { Hemolytic anemia, thrombocytopenia, lymphadenopathy, }(\mathrm{S}) \\
\text { urticarial rashes, hepatosplenomegaly }\end{array}$ \\
\hline P11 & Male & 4 & AlHA, thrombocytopenia, lymphadenopathy, Guillain-Barré syndrome \\
\hline
\end{tabular}

$\mathrm{A}(\mathrm{S})$ indicates splenectomy.

affecting the CD95 ECD. To understand the molecular basis of the apoptotic defect associated with CD95 mutations, we investigated the expression and functional interactions of ICD and ECD mutant forms of CD95.

\section{Methods}

Patient ascertainment and study. The index cases, P4-P11, were referred for study to the Hospital for Special Surgery (Cornell University Medical College, New York, New York, USA) with a presumptive clinical diagnosis of Canale-Smith syndrome (CSS). Clinical data for each of the index cases, firstdegree relatives and any family members reported to have CSS (or another autoimmune disease), was gathered by direct interview, chart review, and verification of data by the physician or pathologist concerned following consent by a institutionally approved protocol. Individuals were classified as "affected" if they satisfied two of the following three criteria: lymphoaccumulation (chronic lymphadenopathy or splenomegaly), systemic autoimmunity, or a CD95 mutation. The extended pedigree data for the P8 family was derived from Rao et al. (25), where the mother of P8 has the pedigree nomination, V-73.

Patients 1 and 2 in this report correspond to patients P1 and P2, originally reported in Drappa et al. (16), who have a CD95 mutation causing a truncation at residue 230 (K230X) or an amino acid substitution at residue 244 (D244Y), respectively. P4-P11 also had an increase in circulating CD3 ${ }^{+}, \mathrm{CD}^{-}$, and $\mathrm{CD}^{-} \mathrm{T}$ cells, defective CD95-mediated apoptosis ( $<40 \%$ of control), lymphadenopathy, splenomegaly, and systemic autoimmunity. A detailed clinical description of these patients is given elsewhere (Vaishnaw, A.K., et al., manuscript in preparation).

Mutation detection. RNA was isolated from activated peripheral blood mononuclear cells (PBMC) and cDNA encoding CD95 prepared by reverse transcriptase (RT)-PCR using primers 01 and 04 , as described previously (16). The PCR products were subcloned into the EcoRI-XhoI sites of the expression vector, pCDNA3 (Invitrogen Corp., Carlsbad, California, USA), and six independent clones for each patient were sequenced using CD95-specific primers (16). CD95L cDNA was amplified by RT-PCR (primer pairs 142F/507R and 448F/1011R) and PCR products were sequenced, as before (16).

All CD95 mutations were verified by PCR amplification of the corresponding exon from genomic DNA and sequencing. The following exons were amplified using the primer pairs and conditions indicated: exon 2 (' GGTGGGTTACACTGGTTTACA;' GTGCTACTCCTAACTGTGAC; $95^{\circ} \mathrm{C} 1 \mathrm{~min}, 55^{\circ} \mathrm{C} 1 \mathrm{~min}$, $72^{\circ} \mathrm{C} 1 \mathrm{~min}, 33$ cycles), exon 7 (' GCATTCTACAAGGCTGAGA; ' CTGCAGTGAGCCATGATT; $95^{\circ} \mathrm{C} 1 \mathrm{~min}, 55^{\circ} \mathrm{C} 1 \mathrm{~min}, 72^{\circ} \mathrm{C}$ 1 min, 33 cycles), and exon 9' CATGGTTTTCACTAATGGGA; ' CACTCTAGACCAAGCTTTGG; $95^{\circ} \mathrm{C} 1 \mathrm{~min}, 55^{\circ} \mathrm{C} 1 \mathrm{~min}$, $72^{\circ} \mathrm{C} 1 \mathrm{~min}, 33$ cycles). Genomic exon-specific single-stranded conformational polymorphism (SSCP) for exons 2,7 , and 9 was carried out as described previously (16), using the appropriate primer pairs (see above and exon 9 primer pair 17/47 in ref. 16).

Preparation of expression constructs and recombinant proteins. The wild-type and mutant CD95 constructs in PCDNA3 were generated as described above. Flag-FADD was prepared by PCR amplification from pCDNA3-AU1-FADD (courtesy of Vishva Dixit, University of Michigan, Ann Arbor, Michigan, USA), and the product was subcloned into pFlag-CMV-2 (Kodak, Rochester, New York, USA). Wild-type, P4, P6, CRD1, and CRD2 CD95-Fc fusion proteins were prepared as described elsewhere (1). The extracellular domain coding region for the P6 CD95 receptor was amplified from the P6pCDNA3 using a $5^{\prime}$ primer (5'-CCGGTACCCCACCATGCTGGGCAT-CTGGA-3') and a $3^{\prime}$ primer (5'-CCGGATCCGCTGGCGAATT-GGCCATTGGCC-3'), and subcloned into pCDNA3-Fc (1). Subsequent CD95-Fc fusion protein expression and purification was as described (1). All expression constructs were verified by automated sequencing before they were used. Rous sarcoma virus (RSV)-Luc (courtesy of Yixin Li, Hospital for Special Surgery, Cornell University Medical College, New York, New York, USA) contains the RSV promoter upstream of firefly luciferase.

Cell culture and transfection. T cells obtained from peripheral blood were activated with anti-CD3 and interleukin-2 (IL2 ), as described previously (16). Adherent human embryonic kidney 293T and COS-1 cells were cultured in DMEM plus $10 \% \mathrm{FCS}$ at $37^{\circ} \mathrm{C}$ in $5 \% \mathrm{CO}_{2}$ with penicillin-streptomycin and Fungizone. Jurkat $\mathrm{T}$ leukemia cells were cultured in Roswell Park Memorial Institute medium (RPMI-1640) plus $10 \% \mathrm{FCS}$ at $37^{\circ} \mathrm{C}$ in $5 \% \mathrm{CO}_{2}$ with penicillin-streptomycin (Gibco/BRL, Gaithersburg, Maryland, USA). Adherent cells were transiently transfected using the calcium phosphate method with $1-4 \mu \mathrm{g}$ plasmid DNA in $60-\mathrm{mm}$ wide tissue culture dishes. Jurkat cells were transfected with $15 \mu \mathrm{g}$ DNA (10 $\mu \mathrm{g}$ CD95 expression vector, $5 \mu \mathrm{g}$ RSV-Luc) by electroporation at $320 \mathrm{~V} / 960 \mu \mathrm{F}$ (Gene Pulser; Bio-Rad Laboratories Inc., Hercules, California, USA). Cells were analyzed $48 \mathrm{~h}$ after transfection. 

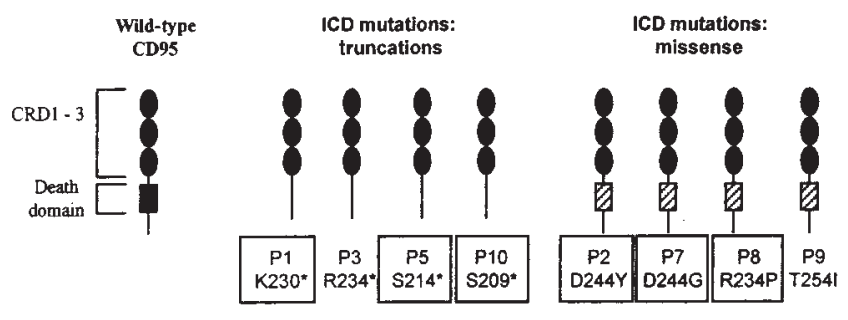

Flow cytometry. The following monoclonal anti-CD95 antibodies were used for flow cytometry (FACScan; Becton Dickinson Immunocytometry Systems, San Jose, California, USA) analysis: UB2 (Immunotech, Westbrook, Maine, USA); DX2 (courtesy of Lewis Lanier, DNAX, palo Alto, California, USA.); anti-Apo-1 (26); CH11 (Upstate Biotechnologies Inc., Lake Placid, New York, USA). A newly generated anti-CD95 monoclonal antibody (MAB), CLB-CD95/2 (27), was kindly provided by L. Aarden (Central Laboratory of Netherlands Red Cross Blood Transfusion Service, Amsterdam, Holland). Nok-1 (PharMingen, San Diego, California, USA) was used for detection of CD95L.

$m R N A$ and protein analysis. RNA was isolated from transfected cells and analyzed by Northern blot assay, as described elsewhere (28). For protein assays, cells were harvested by centrifugation and lysed in NP-40 lysis buffer (1\% NP-40, $20 \mathrm{mM}$ Tris [pH 8.0], $200 \mathrm{mM} \mathrm{NaCl}, 1 \mathrm{mM}$ EDTA, $2 \mathrm{mg} / \mathrm{ml}$ aprotinin, $1 \mathrm{mg} / \mathrm{ml}$ leupeptin, and $25 \mathrm{mg} / \mathrm{ml}$ PMSF) at room temperature. For immunoprecipitation studies, lysate volumes were adjusted to $0.5 \mathrm{ml}$ and then incubated with either IgG $\mathrm{G}_{3}$ anti-Apo- 1 at $1 \mu \mathrm{g} / \mathrm{ml}$ followed by $10 \mu \mathrm{l}$ Sepharose-protein A or $8 \mu \mathrm{l}$ of antiFlag resin $(1: 8 \mathrm{vol} / \mathrm{vol})$. The matrix was washed with RIPA buffer (1\% Triton X-100, $1 \%$ sodium deoxycholate, $0.1 \%$ SDS, $20 \mathrm{mM}$ Tris [pH 7.4], and $150 \mathrm{mM} \mathrm{NaCl}$ ), and the immunoprecipitates were analyzed by SDS-PAGE and Western blotting as described previously (1). Antibodies used included: antiCD95, N18 (Santa Cruz Biotechnology Inc., Santa Cruz, California, USA); anti-Flag, M2 (Kodak); and anti-CD95L, Q20 (Santa Cruz Biotechnology Inc.). For dot-blot assays, cell culture supernates (in serum-free medium) were directly applied to polyvinylidene fluoride (PVDF)-Immobilon-PSQ (Millipore Corp., Bedford, Massachusetts, USA). Membranes were blocked in $5 \%$ milk and sequentially probed with anti-CD95 (N18 or MAB CLB-CD95/2) appropriate horseradish peroxidase (HRP)coupled secondary antibody and processed using enhanced chemiluminescence (ECL) reagents (Pierce Chemical Co., Rockford, Illinois, USA). For immunofluorescence analysis, cells were fixed in $4 \%$ paraformaldehyde plus $0.1 \%$ Triton X-100, blocked with $10 \%$ goat serum/PBS, and then incubated with

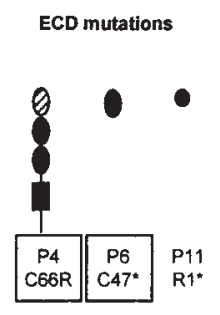

Figure 1

Schematic of the predicted protein structure encoded

by the CD95 mutant alleles from CSS patients, P1-P11. Extracellular cysteine-rich domains (CRD 1-3) and the intracellular death domain are indicated for wild-type CD95. Truncations mutations are indicated by loss of the corresponding domain(s) and missense mutations

" by a hatched domain. Mutant alleles used for in vitro analyses are boxed. CSS, Canale-Smith syndrome; ICD intracellular domain; $E C D$, extracellular domain.
anti-CD95 followed by a 1:100 dilution of FITC-conjugated secondary antibody at $4^{\circ} \mathrm{C}$. The cells were washed and photographed by phase or fluorescence microscopy.

Secondary structure predictions were performed via the following website, http://bioneer.kaist.ac.kr/Pedro/rt_all.html, using the nnpredict and nnsp programs as directed.

Apoptosis assays. Jurkat T-cell apoptosis was induced either by $50 \mathrm{ng} / \mathrm{ml}$ anti-CD95 MAB, CH-11, or CHO-SFMII medium containing soluble CD95L (29). For cells transfected with RSVLuc, 20- $\mu \mathrm{l}$ aliquots of lysed cells were incubated with $100 \mu \mathrm{l}$ luciferase substrate reagent (Promega Corp., Madison, Wisconsin, USA) and analyzed by luminometry.

\section{Results}

Clinical features of CSS patients. Patients 1-3 have been described elsewhere (16). CSS was diagnosed in P4-P11 on the basis of a chronic disease of early childhood characterized by lymphoaccumulation and systemic autoimmunity (15). The clinical features of the eight probands and other affected family members are summarized in Table 1. All developed disease within the first four years of life. Family histories of CSS index cases revealed that P8, P9, and P10 had additional affected members, suggesting an autosomal dominant pattern of inheritance (see below). A detailed clinical description of these patients is presented elsewhere (Vaishnaw, A.K., et al, manuscript in preparation).

CD95/CD95L mutational studies. All patients had heterozygous CD95 mutations as summarized in Table 2 and shown schematically in Fig. 1. Most patients had mutations of the CD95 ICD, which were either frameshift-associated with premature termination (P5 S214X, P10 S209X) or missense mutations (P7 D244G, P8 R234P, P9 T254I). For both P5 and P10, the frameshifts were associated with consensus splice-donor or acceptor mutations. PCR amplification and sequenc-

Table 2

Heterozygous CD95 mutations in patients with the Canale-Smith syndrome

\begin{tabular}{|c|c|c|c|}
\hline Patient & Mutant allele & Protein & Nomination \\
\hline P4 & Codon 66 TGC $\rightarrow$ CGC & Cys $\rightarrow$ Arg & C66R \\
\hline P5 & Intron 8, premature splice acceptor: ctagttttagATGTTA & Frameshift, termination at position 214 & S214X \\
\hline P7 & Codon 244 GAT $\rightarrow$ GGT & Asp $\rightarrow$ Gly & D244G \\
\hline P8 & Codon $234 \mathrm{CGA} \rightarrow \mathrm{CCA}$ & Arg $\rightarrow$ Pro & R234P \\
\hline P9 & Codon 254 ACA $\rightarrow$ ATA & Thr $\rightarrow$ Ile & $\mathrm{T} 254 \mathrm{I}$ \\
\hline P11 & Nucleotide $240 \rightarrow 241$ (GC deletion) & Frameshift, termination at position 1 & $\mathrm{R} 1 \mathrm{X}$ \\
\hline
\end{tabular}

AIntronic residues are in lower case; mutated nucleotide is in bold, italicized, and underlined. 

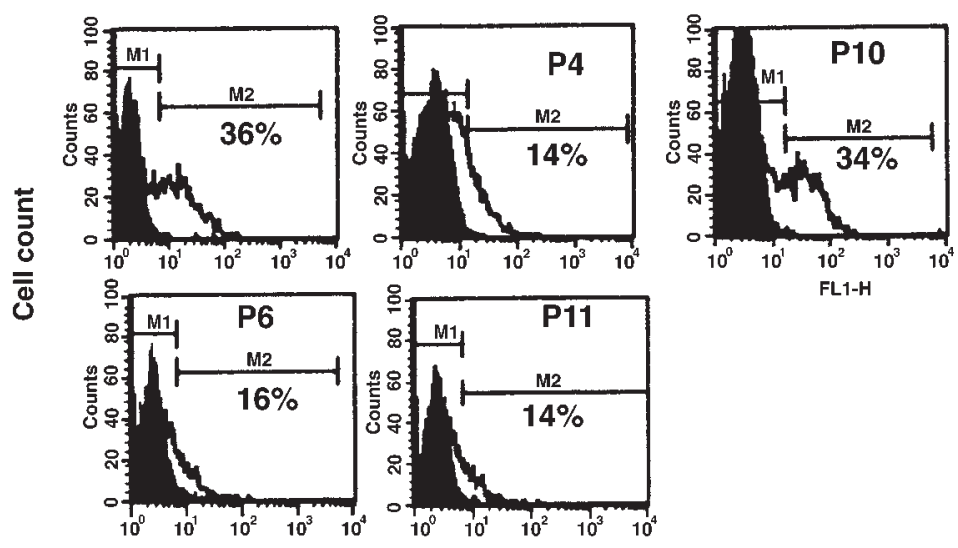

Figure 2

In vivo expression of ECD mutant alleles. PBMC were isolated from patients P4, P6, P10, and P11, and CD95 expression was quantified by flow cytometry using the anti-CD95 MAB, UB2. PBMC staining with an isotype control antibody is indicated by shaded histograms. The percent of CD95 cells for each individual is shown. The corresponding mean channel fluorescence results were as follows: P4 (18.2), P6 (17.1), P10 (34.2), and P11 (16.8). PBMC, peripheral blood mononuclear cells; $M A B$, monoclonal antibody. ing of exon 9 and flanking sequences revealed that P5 had an intron 8 mutation that created a premature splice acceptor site (ctatttttagATGTT $\rightarrow$ ctagttttagATGTT). Similar exon 7 studies confirmed that $\mathrm{P} 10$ had an intron 7 splice-donor mutation (TCCTgtaggt $\rightarrow$ TCCTgaaggt). P4, P6, and P11 each had a mutation within the CD95 extracellular domain. $\mathrm{P} 4$ had an exon 3 point mutation, resulting in a missense codon (C66R). P6 had an exon 2 insertion that caused a frameshift and premature termination at codon 47 (C47X); P11 had a dinucleotide exon 2 deletion followed by an in-frame stop codon, corresponding to position 1 of the mature CD95 protein (R1X). Patients' cDNA was also analyzed for CD95L mutations, but none were identified.

ICD CD95 mutant proteins are expressed and inbibit apoptosis mediated by wild-type CD95. To examine CD95 expression in CSS patients, freshly isolated PBMC were analyzed by flow cytometry using the anti-CD95-specific MAB, UB2. All individuals with an ICD mutation (P1, P2, P3, P5, P7, P8, P9, and P10) had normal CD95 expression, but expression was reduced in patients with the ECD mutations (P4, P6, P11) (Fig. 2). These findings suggested that the ICD, but not the ECD mutant proteins were coexpressed with wild-type CD95 on the cell surface. To verify this finding, a representative spectrum of ICD (P1, P2, P5, P7, P8, P10) and ECD (P4, P6) mutants were subcloned into the expression vector pCDNA3 and transfected into $293 \mathrm{~T}$ cells. Consistent with findings from patients' PBMC, the wild-type (WT) and ICD, but not the ECD, mutant alleles were readily detected by flow cytometry with the UB2 MAB (Fig. 3a).

To assess the functional impact of a heterozygous CD95 mutation, we next examined CD95 function following transfection of the mutant alleles in Jurkat $\mathrm{T}$ cells, which express CD95 and undergo apoptosis in response to an agonistic anti-CD95 stimulus (30). Jurkat cells were cotransfected with either WT CD95, mutant CD95, or pCDNA3 vector, together with the constitutively active reporter construct, RSV-Luc. Twenty-four hours after transfection, aliquots of transfected cells were incubated with the agonistic anti-CD95 antibody $\mathrm{CH}-11$ or isotype control for an additional 24 hours, and then assayed for luciferase activity. The mean values of two such Jurkat protection assays are shown in Fig. $3 b$. All ICD mutants clearly inhibited CD95-mediated apoptosis, as evidenced by the relatively high cell viability in the presence of CD95 agonist. These results suggested that the ICD mutants act as dominant-negative molecules as also shown for the ICD mutants reported by Fisher et al. (19).

ICD CD95 mutants abrogate FADD recruitment to the death domain. In the presence of CD95L, CD95 aggregation results in the generation of a death signal via the cytoplasmic recruitment of FADD to the death domain of CD95 $(3,4)$. The binding of FADD to WT or ICD
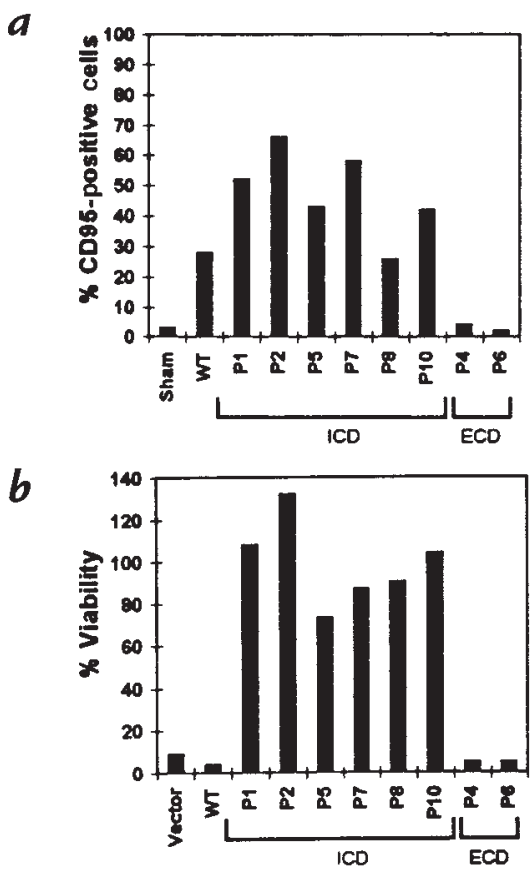

Figure 3

Surface expression and dominant-negative function of ICD mutant CD95 alleles. (a) Expression. Human embryonic kidney 293T cells were transfected with PCDNA3 expression vectors encoding WT or mutant CD95. CD95 expression was analyzed by flow cytometry using the anti-CD95 MAB, UB2. The percent of CD95-positive cells for each transfection is shown. (b) Function. CD95-positive Jurkat $T$ cells were cotransfected with the same panel of expression vectors as in $a$ and with the reporter gene, RSV-Luc. The cells were separated into two aliquots and cultured with either the agonistic anti-CD95 antibody $\mathrm{CH} 11(50 \mathrm{ng} / \mathrm{ml})$ or an isotype control antibody $24 \mathrm{~h}$ after transfection. Luciferase expression was assayed at $48 \mathrm{~h}$ and percent viability was calculated by the following formula: (luciferase activity of $\mathrm{CH} 11$ /luciferase activity of control antibody) $\times 100$. The data shown are the mean results of two independent experiments. WT, wild type; RSV, Rous sarcoma virus. 
mutant CD95 receptors was therefore assessed. 293T cells were cotransfected with Flag-tagged FADD and either WT CD95 or one of the ICD mutants. Cell lysates were prepared and equal amounts of protein analyzed by immunoprecipitation (IP), using the agonistic antiCD95 MAB anti-Apo-1, followed by Western blot analysis with anti-Flag. As shown in Fig. 4a, despite comparable expression of WT or mutant CD95 and Flag-FADD (upper and middle panels), IP with anti-CD95 coprecipitated FADD in cells transfected with WT CD95 but not in cells transfected with an ICD mutant (lower panel).

To approximate the in vivo situation more closely, we next examined FADD recruitment after coexpression of an ICD mutant with WT CD95 and Flag-FADD. 293T cells were transfected with constant amounts of the WT CD95 and Flag-FADD expression constructs, along with increasing amounts of the P10 mutant. Lysates were immunoprecipitated with anti-Apo-1 and analyzed by Western blot. As shown in Fig. 4b, the amount of FADD recruited to the complex diminished with increasing P10 expression. At approximately equivalent WT and mutant CD95 expression levels, FADD binding was undetectable, providing a molecular basis for the dominant-negative action of ICD mutant alleles.

Structural distortions of the death domain secondary to ICD mutations. Nuclear magnetic resonance (NMR) spectroscopy has shown that the CD95 death domain is composed of six $\alpha$-helices ( $\alpha 1$ to $\alpha 6$ ), which are crucial for homotypic death-domain interactions (31). To understand the impact of the ICD mutations (P2, P7, P8) on the helical structure of the death domain, we undertook a secondary structure analysis using nnpredict and nnssp, as described in Methods. Both nnpredict and nnssp modeled the peptide sequence for WT CD95 (residues 223-273) into three discrete $\alpha$-helical domains, corresponding closely to $\alpha 2, \alpha 3$, and $\alpha 4$ from the studies of Huang et al. (31). When ICD mutants were analyzed, nnpredict and nnsp predicted that P7 D244G caused a break in the $\alpha 3$ helix and that P8 R234P had a similar effect on the $\alpha 2$ helix (Fig. 5). These data provide a structural basis for the failure of the these ICD mutants to bind FADD (Fig. 4). Unlike P7 D244G, the P2 missense mutation D244Y was not predicted to cause an alteration of the $\alpha 3$ helix. Because D244 is a charged surface residue on the surface of the death domain (31), P2 D244Y may have a direct effect on FADD binding.

Expression of ECD CD95 mutant proteins. In contrast to CD95 expression on PBMC from patients with ICD mutations, CD95 expression was significantly lower on $\mathrm{T}$ cells of P4, P6, and P11 when analyzed by the MAB UB2 (Fig. 2) and was undetectable on 293T cells transfected with the corresponding CD95 expression vectors (Fig. $3 a$ ). Reduced expression of CD95 in P6 and P11 was expected, because these mutations are predicted to lead to severely truncated peptides (Fig. 1), whereas P4 contains only a single point mutation at residue 66 (Table 2).

To define the mechanisms responsible for alteration in CD95 expression and function in these patients, the mutant cDNAs were transfected into 293T and Jurkat T cells. Northern blot analysis revealed that equivalent levels of CD95 mRNA were expressed in all transfectants (data not shown). None of the anti-CD95 antibodies-

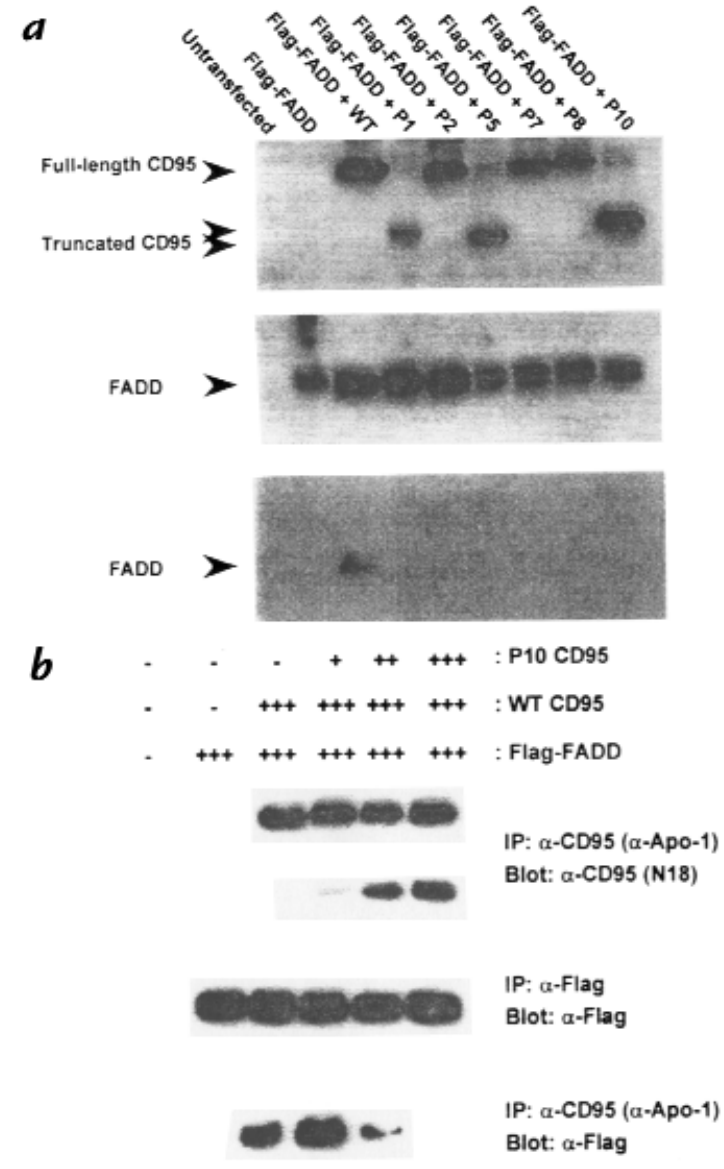

Figure 4

Defective protein-protein interactions of ICD CD95 mutant alleles. (a) ICD CD95 mutant alleles fail to recruit FADD to the CD95 death domain. 293T cells were cotransfected with pCDNA3 expression vectors for WT or mutant CD95 and Flag-FADD in pFlag-CMV-2. Transfected cells were lysed and subjected to IP-Western blot using the antibodies indicated in the figure. The trace signals of WT CD95 in lanes P1, P5, and P10 are due to slight spillover from adjacent lanes and were not seen when these mutants were run separately. (b) The dominant-negative action of ICD mutant CD95 is dose-dependent. 293T cells were transiently cotransfected with expression vectors for Flag-FADD and WT or P10 mutant CD95. Transfections were carried out using constant amounts of total DNA (4 $\mu \mathrm{g})$ using the pCDNA vector DNA, WT CD95 $(0.5 \mu \mathrm{g})$ and Flag-FADD $(0.5 \mu \mathrm{g})$ DNA, but the amount of DNA for the ICD mutant P10, was increased between experiments (lanes 4-6; + $[0.5 \mu \mathrm{g}]$ to $+++[1.5 \mu \mathrm{g}]$ ). The total amount of DNA transfected was kept constant. Transfected cells were lysed and analyzed by immunoprecipitation followed by Western blotting using the antibodies indicated. IP, immunoprecipitation.

UB2, CH11, anti-Apo-1, or DX2- stained 293T cells transfected with P4 or P6 by flow cytometry. However, the MAB CLB-CD95/2 stained P4 transfected cells, albeit less intensely than wild type (Fig. 6a). Because CLB-95/2 binds to CRD2 of CD95 (Van Lopik, T., Vaishnaw, A. K., and Aarden, L., unpublished observations), we conclude that the P4 CRD1 mutation (C66R) substantially distorts the structure of both CRD1 and CRD2.

The P6 mutation predicted the synthesis of a 46-amino acid peptide, truncated in the CRD1 of CD95. To determine whether this peptide was secreted, $293 \mathrm{~T}$ cells were transfected with expression vectors for 


\begin{tabular}{|c|c|c|}
\hline & $\alpha 2$ & $\alpha \mathbf{3}$ \\
\hline CD95 & - - -ннннН & 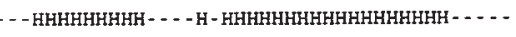 \\
\hline $23-273$ & MTLSQVKG & RKNGVNEAKIDEI KNDNVQDTAEQKVQLLRNWHQLHGKKEA \\
\hline $\mathbf{P} 2$ & - - НнННН & 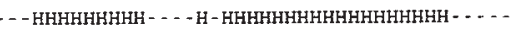 \\
\hline D244Y & MTLSQVKG & KNGVNEAKI I E I KNDNVQDTAEQKVQLLRNWHQLHGKKEA \\
\hline P7 & - - - HНHHF & 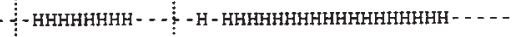 \\
\hline D244G & MTLSQVKG & NGVNEAKIGEIKNDNVQDTAEQKVQLLRNWHQLHGKKEA \\
\hline P8 & - - HHH - & 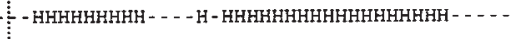 \\
\hline R234P & MLSQVK & KNGVNEAKIDEI KNDNVQDTAEQKVQLLRNWHQLHGKKEA \\
\hline
\end{tabular}

\section{Figure 5}

Death domain missense mutations (P7 and P8) distort its $\alpha$-helical structure. The peptide sequence for wild-type CD95 (residues 223-273) and the same regions from P2, P7, and P8 mutant alleles were analyzed by secondary structure predictive models (see Methods). The regions of each sequence that were predicted to adopt an $\alpha$-helical conformation $(H)$ are indicated above the corresponding region of the amino acid sequence. Dashes (-) represent areas predicted not to form an $\alpha$-helix. The stippled boxes highlight the regions predicted to be altered by the mutations.

P6 or TM (WT CD95 lacking the transmembrane domain), or with pCDNA3 alone. Cell supernatants were analyzed by dot-blot using the $\mathrm{NH}_{2}$-terminus-specific antibody N18. While TM was readily detected in this assay, P6 was not (data not shown). Because immunofluorescence revealed intense intracellular staining for WT CD95 but no staining for P6 (not shown), these results suggested that the P6 ECD mutant was degraded intracellularly. A similar result was expected for P11, which is truncated immediately after the signal peptide.

Apoptosis in ECD mutants. The findings so far indicated that partial loss of CD95 function in P6 and 11 could be explained by lack of expression of the mutant proteins and that defective CD95 function of the P4 mutant resulted from failure to bind CD95 ligand (1). However, the apoptotic defect in the patients' own cells was greater than $50 \%$ (16). To determine whether the ECD mutants exerted a protective effect against authentic CD95 ligand, Jurkat cells were transfected with the two ECD mutants and used as targets for 293T cells expressing CD95L (29) in a coculture experiment. As shown in Fig. $7 a$, P10 transfected Jurkat T cells were protected from CD95L-mediated apoptosis, but those transfected with P4, P6, or WT CD95 were as susceptible to apoptosis as pCDNA3-transfected Jurkat cells. It has been reported previously that short ECD-truncation mutants could be secreted and inhibit anti-CD95-mediated apoptosis (32). We therefore tested the ability of supernatants from activated T cells obtained from P6 to inhibit apoptosis. As shown in Fig. $7 b$, the culture supernates failed to inhibit anti-CD95-mediated apoptosis, even when added undiluted. A similar lack of inhibition was observed when supernates from P6 pCDNA3 transfected 293T cells were used (not shown). Finally, because P4 and P6 CD95-Fc fusion proteins also failed to bind and inhibit CD95 ligands (Fig. 7c), the ECD mutants appear to exert their effect solely by loss of function.

ICD mutations are bighly penetrant in CSS families. Because ICD mutants exhibited a dominant-negative action, we hypothesized that they may have higher penetrance than ECD mutations. Clinical histories and
CD95 genotypes were therefore obtained from the firstdegree relatives of patients P1-P11 (except for P1 and P7, which arose spontaneously). Families of ICDmutant patients P2, P8, P9, and P10 each had additional member(s) with the CSS phenotype (Fig. 8). When the proportion of nonindex cases with an ICD CD95 mutation were determined, 60\% (6/10) were affected. In contrast, the index cases P4, P6, and P11 were the only affected members in ECD-mutation families, and none of the first-degree relatives with a CD95 mutation expressed the CSS phenotype (0/5).

\section{Discussion}

CD95 mutations in mice and humans are associated with defective lymphocyte apoptosis and autoimmunity. The precise molecular mechanism(s) by which these mutations cause reduced apoptosis has not been defined previously. Gene mutations may affect mRNA or protein expression, stability or function. In this study, we examined the molecular mechanisms responsible for loss of CD95-mediated apoptosis, using mutant alleles cloned from eight different patients with heterozygous CD95 mutations. The mutations, affecting either the ICD (P1, P2, P5, P7, P8, P10) or ECD (P4, P6, P11) of CD95, were all associated with markedly reduced CD95-mediated apoptosis of activated T cells. However, different mechanisms appeared to be responsible for the defective apoptosis associated with ICD versus ECD mutations.

ICD mutant alleles were readily detected at the cell surface, indicating that they did not have an appreciable effect on protein expression. However, when they were transfected into Jurkat $\mathrm{T}$ cells, which constitutively express WT CD95 and are highly sensitive to CD95-mediated apoptosis (30), all of the ICD mutants caused a striking reduction in CD95-mediated apoptosis. These data confirm findings from functional studies of similar CD95

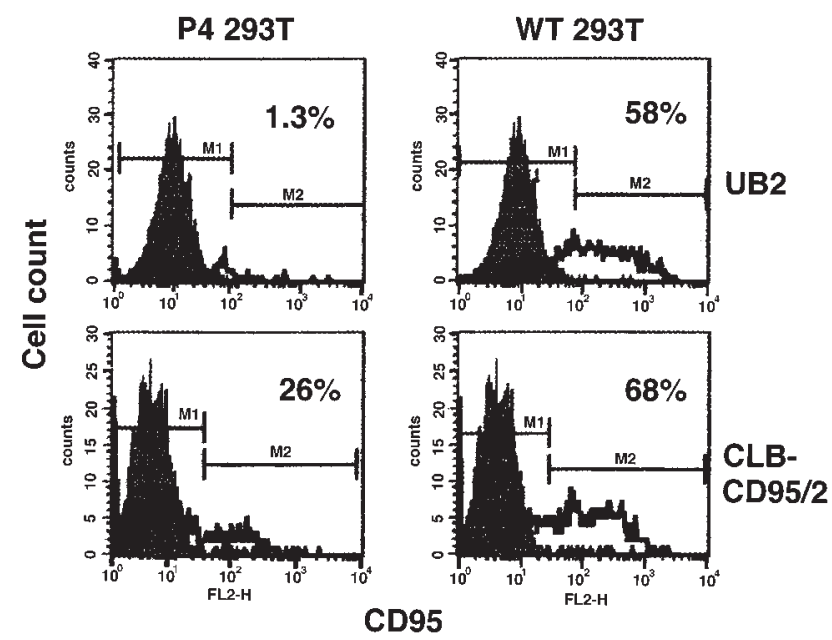

Figure 6

CD95 expression for ECD mutation patients P4 and P6 293T cells were transfected with expression vectors for P4 or WT CD95. Transfected cells were analyzed by flow cytometry using UB2 or CLB-CD95/2. For each transfectant, the percent of CD95-positive cells is indicated, with shaded histograms representing staining of PCDNA3 (vector control) transfected cells. The results shown are representative of three experiments. 
mutations in other patients (19). Ligand-induced clustering of CD95 recruits the adapter molecule, FADD, to the intracytoplasmic death domain of $\operatorname{CD} 95(3,4)$. This results in the recruitment and activation of FLICE/Mach/caspase-8 $(33,34)$ and FLICE2/caspase-10 (35), initiating sequential caspase activation, intracellular proteolysis, and cell death. We therefore examined the ability of CD95-mutant proteins to recruit FADD, by cotransfection and IP assays using 293T cells, which do not constitutively express CD95. P1, P5, and P10 have truncations of the CD95 death domain, and failure of these mutant proteins to bind FADD was anticipated. Alleles P2 (D244Y), P7 (D244G), and P8 (R234P) had amino acid substitutions of highly conserved residues in subdomains $\alpha 2$ (R234P) and $\alpha 3$ (D244Y, D244G) of the death domain. It is known that the amino acids glycine or proline within a peptide subdomain greatly reduce the probability of $\alpha$-helix formation $(36,37)$. Secondary structure analysis predicted that the death domain substitutions, R234P and D244G, broke the $\alpha 2$ and A3 helices respectively, whereas D244Y did not. Because D244 is solvent-accessible and forms part of the interface involved in CD95 protein-protein interaction (31), D244 may be a necessary contact residue for FADD binding. When P2, P7, or P8 were coexpressed with FADD, they all failed to recruit FADD. These findings were consistent with the secondary structure predictions and with previous alanine-scanning mutagenesis of the CD95 death domain, which revealed that $\mathrm{R} 234 \mathrm{~A}$ or D244A abolished both CD95-CD95 and CD95-FADD death-domain interactions in vitro (31).

To determine whether the ICD mutants could prevent binding of FADD to heterotypic aggregates of mutant and wild-type CD95, we titrated mutant CD95 against a constant amount of WT CD95 in a cotransfection study. Co-IP of P10 and WT CD95 demonstrated that the mutant caused a dose-dependent inhibition of FADD binding to the receptor complex. Knockout studies have shown that FADD is absolutely required for CD95-mediated apoptosis $(38,39)$, so these observations provide a sufficient explanation for the loss of CD95-mediated apoptosis in patients with ICD mutations.

Of the three ECD mutants - P4, P6, and P11 - two (P6, P11) cause premature truncation within the first 50 amino acids of the protein and were not expressed on the cell surface. Indeed, we found little or no intracellular expression of the $\mathrm{P} 6$ peptide in transfectants, suggesting that it was rapidly degraded. Although cell-surface expression of the P4 mutant could not be detected by a panel of anti-CD95 monoclonal antibodies that bind to CRD1, CLB-95/2, a CRD2-specific antibody, revealed cell-surface expression of P4. Whereas the absolute intensity of staining was reduced compared to WT CD95, the relative intensity of intracellular to extracellular staining was similar to that observed with WT CD95 (A.K. Vaishnaw and K.B. Elkon, unpublished observations). These findings suggest that reduced intensity of staining is explained by alteration in the conformation of CRD1 and CRD2 caused by substitution of cysteine at residue 66 . Reduction of cysteine residues in the ECD of CD95 has been shown to markedly attenuate anti-CD95 MAB binding (40), and we have shown previously that the C66R struc- $a$

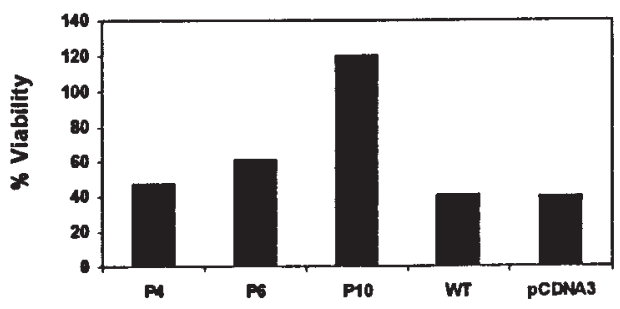

$b$

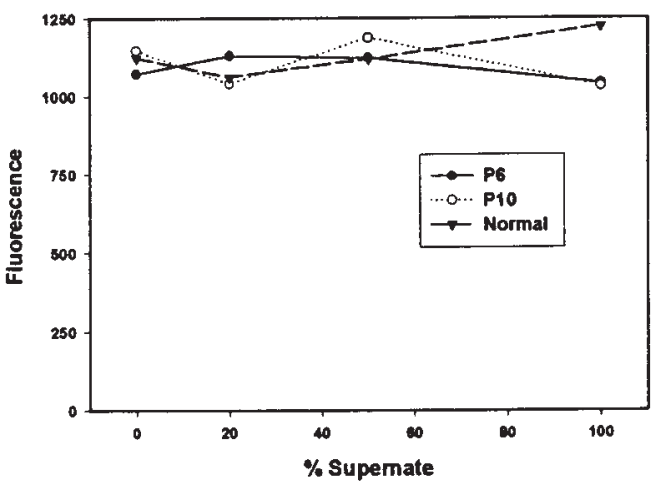

C

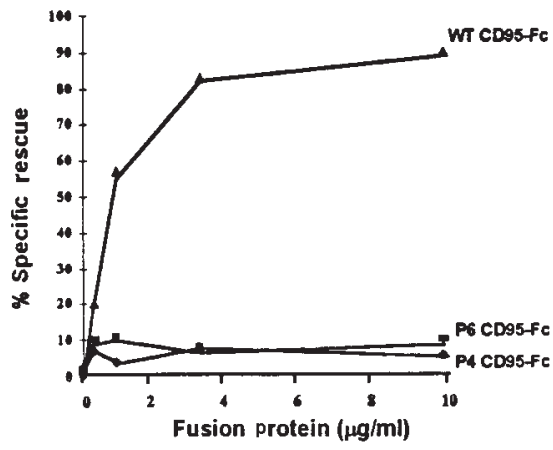

Figure 7

The ECD mutants, P4 and P6, do not exert a dominant-negative effect and do not inhibit CD95L function. (a) Direct transfection assay. Jurkat $T$ cells were cotransfected with RSV-Luc and P4, P6, P10, WT CD95, or PCDNA3. Half of the transfected cells were applied to monolayers of 293T cells expressing WT CD95 L and half to gld CD95L, where gld represents the inactive CD95L mutant $24 \mathrm{~h}$ after transfection (29). At $48 \mathrm{~h}$, the Jurkat cells were harvested, and the ratio of luciferase activity [(WT CD95L/gld CD95L) $\times$ 100 ] was calculated. (b) Supernate transfer assay. $T$ cells from a normal control, P6, or P10 were activated with anti-CD3 and IL-2 for 7 days. The supernates from these cultures were added in the proportions shown on the $x$-axis to 50,000 Jurkat cells. After the addition of $50 \mathrm{ng} / \mathrm{ml}$ of anti-CD95, viability was assessed in triplicate by the Alamar blue assay at $24 \mathrm{~h}$, and the results were expressed by the fluorescence emission at $590 \mathrm{~nm}$. (c) CD95 ligand binding assay. Jurkat $T$ cells were incubated with the CD95-Fc fusion proteins: WT, P4, and P6 $(0.1-10 \mathrm{mg} / \mathrm{ml})$ in the presence of recombinant soluble CD95L. The percent specific rescue from apoptosis associated with each protein is shown. The experiments shown in a-c were performed on at least two separate occasions with virtually identical results. Greater than $100 \%$ viability is most likely explained by the costimulatory effect of CD95 signaling in the absence of apoptosis (44). IL, interleukin.

tural alteration leads to loss of binding to CD95L (1).

Despite the evidence for lack of expression of P6, we examined whether ECD mutants could interfere with the function of the wild-type allele. Neither of the patients who have ECD mutants, P4 or P6, had a dominant-negative effect on wild-type CD95 function in Jurkat T cells. Cascino et al. (32) have, however, reported that activated $\mathrm{T}$ 
ICD
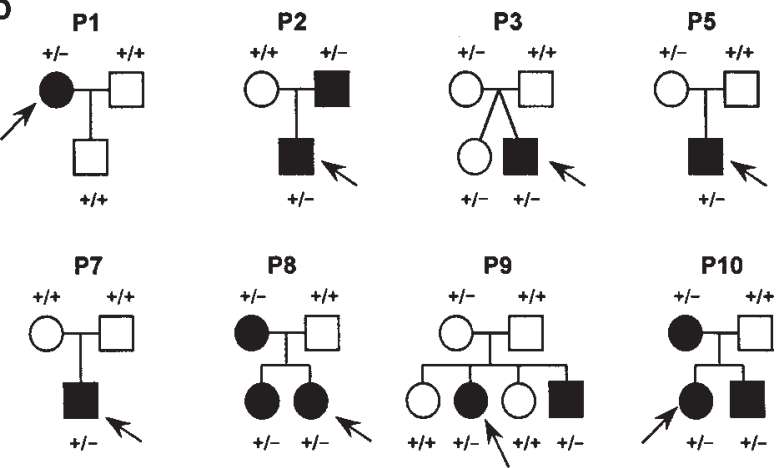

ECD
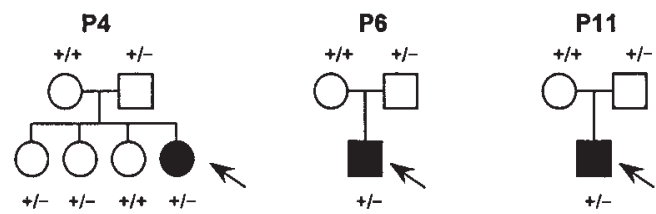

Figure 8

Penetrance analysis of CSS families P1-P11. The nuclear families for index cases P1-P11 (arrows) are shown. CSS affected are noted by shaded symbols. CD95 genotypes were determined by SSCP and are represented as +/- (heterozygous mutation) or +/+ (WT CD95). Parents of P1 are not shown, but have been genotyped previously, and both are $+/+(16)$. Overall, $77 \%(14 / 18)$ individuals with an ICD mutation and $37 \%(3 / 8)$ of ECD mutation carriers had CSS. Considering only nonindex cases, to correct for ascertainment bias, penetrance was 6/10 (ICD) and 0/5 (ECD). SSCP, single-stranded conformational polymorphism.

cells can secrete alternatively spliced CD95 variants that are lacking the transmembrane domain and that are truncated in CRD1, -2 , and/or -3 . The products of these alternatively spliced forms apparently inhibited anti-CD95 mediated apoptosis. In our studies, supernatants from P6 transfected cells failed to block CD95L-mediated apoptosis, and neither the P4 nor P6 CD95-Fc proteins bound CD95L. Because the P11 mutation causes a truncation immediately after the signal peptide, it is difficult to conceive of any interfering mechanism, even if the peptide is synthesized. In sum, all of the evidence indicates that the ECD mutations are associated with a loss of function rather than a dominant-negative mode of action.

Although lymphadenopathy frequently becomes less severe with age $(16,22)$, conceivably leading to underestimation of phenotype in parents of those affected, we compared the penetrance of CSS in families with ICD and ECD mutations. Consistent with our results demonstrating that ICD mutations were dominant-negative, we observed that these alleles were associated with a more highly penetrant CSS syndrome. Analysis of the extended pedigrees for $\mathrm{P} 8, \mathrm{P} 9$, and $\mathrm{P} 10$ revealed that $\mathrm{P} 8$ and P9 also had additional individuals outside the nuclear families who were affected with a CSS-like syndrome (Vaishnaw, A.K., et al., manuscript in preparation). Indeed, for P8, the family history for CSS was striking, and the pedigree had been reported previously as containing seven individuals with an autosomal dominant form of "idiopathic hereditary splenomegaly and hypersplenism” (25). In contrast to ICD mutations, families with an ECD mutation had only one individual affected with CSS, although other family members carried the mutation. In the $\mathrm{P} 4$ pedigree, two siblings and the father of the index case were unaffected carriers, and the father, two paternal uncles, and the paternal grandfather of P6 were all unaffected carriers of an ECD mutation.

Although it is clear that ICD mutations are not always associated with CSS, previous reports of ICD and a small number of ECD mutations are consistent with reduced penetrance of ECD mutations (16, 18, 19, 21-24). Only one family has been reported with an ECD mutation and multiple affected members, but all of these were compound heterozygotes for an ECD and an ICD mutation (21). These observations are similar to studies of heterozygous CD95 mutations in mice, in which lprcg (ICD mutation) shows greater penetrance than the $l p r$ allele (loss of function mutation) (41-43). Together, these findings demonstrate a remarkable complexity in the nature, penetrance, mechanisms of action, and clinical expression of CD95 mutations in the outbred human populations.

\section{Acknowledgments}

We thank Nathan Brot for helpful discussions. Supported, in part, by grants from the National Institutes of Health (AR 45482) and the Histiocytosis Association of America. A.K. Vaishnaw is a fellow of theSLE Foundation, New York and was supported, in part, by the Arthritis and Rheumatism Council, United Kingdom.

1. Orlinick, J.R., Vaishnaw, A.K., Elkon, K.B., and Chao, M.V. 1997. Requirement of cysteine-rich repeats of the Fas receptor for binding by the Fas ligand. J. Biol. Chem. 272:28889-28894.

2. Nagata, S. 1997. Apoptosis by death factor. Cell. 88:355-365.

3. Chinnaiyan, A.M., O'Rourke, K., Tewari, M., and Dixit, V.M. 1995. FADD, a novel death domain-containing protein, interacts with the death domain of Fas and initiates apoptosis. Cell. 81:505-512.

4. Boldin, M.P., et al. 1995. A novel protein that interacts with the death domain of Fas/Apo-1 contains a sequence motif related to the death domain. J. Biol. Chem. 270:7795-7798.

5. Salvesen, G.S., and Dixit, V.M. 1997. Caspases: intracellular signaling by proteolysis. Cell. 14:443-446.

6. Tepper, C.G., et al. 1995. Role for ceramide as an endogenous mediator of Fas-induced cytotoxicity. Proc. Natl. Acad. Sci. USA. 92:8443-8447.

7. Cifone, M.G., et al. 1994. Apoptotic signaling through CD95 (Fas/Apo1) activates an acidic sphingomyelinase. J. Exp. Med. 177:1547-1552.

8. Santana, P., et al. 1996. Acid sphingomyelinase-deficient human lymphoblasts and mice are defective in radiation-induced apoptosis. Cell. 86:189-199.

9. Yang, X., Khosravi-Far, R., Chang, H.Y., and Baltimore, D. 1997. Daxx, a novel Fas-binding protein that activates JNK and apoptosis. Cell. 89:1067-1076.

10. Kennedy, N.J., and Budd, R.C. 1998. Phosphorylation of FADD/MORT1 and Fas by kinases that associate with the membrane-proximal cytoplasmic domain of Fas. J. Immunol. 160:4881-4888.

11. Russell, J.H., Rush, B., Weaver, C., and Wang, R. 1993. Mature T cells of autoimmune lpr/lpr mice have a defect in antigen-stimulated suicide. Proc. Natl. Acad. Sci. USA. 90:4409-4413.

12. Singer, G.G., and Abbas, A.K. 1994. The Fas antigen is involved in peripheral but not thymic deletion of $\mathrm{T}$ lymphocytes in $\mathrm{T}$ cell receptor transgenic mice. Immunity. 1:365-371.

13. Rathmell, J.C., Townsend, S.E., Xu, J.C., Flavell, R.A., and Goodnow, C.C. 1996. Expansion or elimination of B cells in vivo: dual roles for CD40and Fas (CD95)-ligands modulated by the B cell antigen receptor. Cell. 57:319-329.

14. Cohen, P.L., and Eisenberg, R.A. 1991. lpr and gld: single gene models of systemic autoimmunity and lymphoproliferative disease. Annu. Rev. Immunol. 9:243-269.

15. Canale, V.C., and Smith, H.C. 1967. Chronic lymphadenopathy simulating malignant lymphoma. J. Pediatr. 70:891-899.

16. Drappa, J., Vaishnaw, A.K., Sullivan, K.E., Chu, J.L., and Elkon, K.B. 1996. The Canale Smith syndrome: an inherited autoimmune disorder associated with defective lymphocyte apoptosis and mutations in the Fas gene. N. Engl. J. Med. 335:1643-1649. 
17. Sneller, M.C., et al. 1992. A novel lymphoproliferative/autoimmune syndrome resembling murine $\mathrm{lpr} / \mathrm{gld}$ disease. J. Clin. Invest. 90:334-341.

18. Rieux-Laucat, F., et al. 1995. Mutations in Fas associated with human lymphoproliferative syndrome and autoimmunity. Science. 268:1347-1349.

19. Fisher, G.H., et al. 1995. Dominant interfering Fas gene mutations impair apoptosis in a human lymphoproliferative syndrome. Cell. 81:935-946.

20. Dianzani, U., et al. 1997. Deficiency of the Fas pathway without Fas gene mutations in pediatric patients with autoimmunity/lymphoproliferation. Blood. 89:2871-2879.

21. Bettinardi, A., et al. 1997. Missense mutations in the Fas gene resulting in autoimmune lymphoproliferative syndrome: A molecular and immunological analysis. Blood. 89:902-909.

22. Sneller, M.C., et al. 1997. Clinical, immunologic, and genetic features of an autoimmune lymphoproliferative syndrome associated with abnormal lymphocyte apoptosis. Blood. 89:1341-1348.

23. Pensati, L., et al. 1997. Fas/Apo1 mutations and autoimmune lymphoproliferative syndrome in a patient with type 2 autoimmune hepatitis. Gastroenterology. 113:1384-1389.

24. Kasahara, Y., et al. 1998. Novel Fas (CD95/APO-1) mutations in infants with a lymphoproliferative disorder. Int. Immunol. 10:195-202.

25. Rao, L. M., Shahidi, N.T., and Opitz, J.M. 1974. Hereditary splenomegaly with hypersplenism. Clin. Genet. 5:379-386.

26. Trauth, B. C., et al. 1989. Monoclonal antibody-mediated tumor regression by apoptosis. Science. 245:301-305.

27. Van Lopik, T., et al. 1999. SLE patients with high plasma levels of sFas are at risk for developing a relapse. J. Rheumatol. In press.

28. Chu, J.L., et al. 1995. Massive upregulation of the Fas ligand in lpr and gld mice. J. Exp. Med. 181:393-398.

29. Orlinick, J.R., Elkon, K.B., and Chao, M.V. 1997. Separate domains of the human Fas ligand dictate self-association and receptor binding. J. Biol. Chem. 272:32221-32229.

30. Dhein, J., Walczak, H., Bäumler, C., Debatin, K.-M., and Krammer, P.H. 1995. Autocrine T cell suicide mediated by APO-1/(Fas/CD95). Nature. 373:438-441.

31. Huang, B., Eberstadt, M., Olejniczak, E., Meadows, R., and Fesik, S. 1996. NMR structure and mutagenesis of the Fas (APO-1/CD95) death domain. Nature. 384:638-641.

32. Cascino, I., Fiucci, G., Papoff, G., and Ruberti, G. 1995. Three function- al soluble forms of the human apoptosis-inducing Fas molecule are produced by alternative splicing. J. Immunol. 154:2706-2713.

33. Boldin, M.P., Goncharov, T.M., Goltsev, Y.V., and Wallach, D. 1996. Involvement of $\mathrm{MACH}$, a novel MORT1/FADD-interacting protease, in Fas/Apo-1 and TNF receptor-induced cell death. Cell. 85:803-815.

34. Muzio, M., et al. 1996. FLICE, a novel FADD-homologous ICE/CED-3like protease, is recruited to the CD95 (Fas/APO-1) death-inducing signaling complex. Cell. 85:817-827.

35. Vincenz, C., and Dixit, V.M. 1997. Fas-associated death domain protein interleukin-1beta- converting enzyme 2 (FLICE2), an ICE/Ced-3 homologue, is proximally involved in CD95- and p55-mediated death signaling. J. Biol. Chem. 7:6578-6583.

36. O'Neil, K.T., and DeGrado, W.F. 1990. A thermodynamic scale for the helix-forming tendencies of the commonly occurring amino acids. Science. 250:646-651.

37. Chakrabartty, A., Schellman, J.A., and Baldwin, R.L. 1991. Large differences in the helix propensities of alanine and glycine. Nature. 351:586-588.

38. Zhang, J., Cado, D., Chen, A., Kabra, N.H., and Winoto, A. 1998. Fasmediated apoptosis and activation-induced $\mathrm{T}$ cell proliferation are defective in mice lacking FADD/Mort1. Nature. 392:296-300.

39. Newton, K., Harris, A.W., Bath, M.L., Smith, K.G.C., and Strasser, A. 1998. A dominant interfering mutant of FADD/MORT1 enhances deletion of autoreactive thymocytes and inhibits proliferation of mature $\mathrm{T}$ lymphocytes. EMBO. J. 17:706-718

40. Fadeel, B., Lindberg, J., Achour, A., and Chiodi, F. 1998. A three-dimensional model of the Fas/Apo-1 molecule: cross-reactivity of anti-Fas antibodies explained by structural mimicry of antigenic sites. Int. Immunol. 10:131-140.

41. Matsuzawa, A., Kimura, M., Muraiso, T., Kominami, R., and Katagiri, T. 1991. Genotype- restricted lymphoproliferation in autoimmune lpr mice. Eur. J. Immunol. 21:1535-1542.

42. Matsuzawa, A., Katagiri, T., Ogata, Y., Kominami, R., and Kimura, M. 1994. Lymphadenopathy induced by the cooperation between $\mathrm{lpr}^{\mathrm{cg}}$ and gld genes is of lpr but not of gld phenotype. Eur. J. Immunol. 24:1714-1716.

43. Ogata, Y., et al. 1993. Distinct expression of $\mathrm{lpr}^{\mathrm{cg}}$ in the heterozygous state on different genetic backgrounds. Cell. Immunol. 148:91-102.

44. Alderson, M.R., et al. 1993. Fas transduces activation signals in normal human T lymphocytes. J. Exp. Med. 178:2231-2235. 